\title{
Properties of flavour-singlet pseudoscalar mesons from lattice QCD
}

\author{
Carsten Urbach ${ }^{1, \star}$ \\ ${ }^{1}$ HISKP (Theorie), Universität Bonn, Nussallee 14-16, 53115 Bonn, Germany
}

\begin{abstract}
We report on the status of the determination of properties of flavour-singlet pseudoscalar mesons using Wilson twisted mass lattice QCD at maximal twist. As part of project $\mathbf{C 7}$, a large number of phenomenologically relevant quantities could be extracted from first principle, from $\eta$ and $\eta^{\prime}$ masses to decay widths of pseudoscalar mesons to two photons.
\end{abstract}

\section{Introduction}

In the early days of the theory of strong interactions, the peculiar mass hierarchy found experimentally for the nine light pseudoscalar mesons led to the formulation of the "U(1) problem" [1]. If also the axial $U(1)$ symmetry was spontaneously broken in the SU(3) chiral limit of QCD, one would expect the ninth pseudoscalar meson to have a mass similar or a bit lighter than the kaons and the $\eta$ meson. The solution to this puzzle comes from the anomalous breaking of the corresponding axial current $[2,3]$

$$
\partial^{\mu} A_{\mu}^{0}=\partial^{\mu}\left(\bar{q} \gamma_{\mu} \gamma_{5} q\right)=\frac{\alpha_{s}}{4 \pi} G \tilde{G}
$$

at the quantum level triggered by a non-trivial topological structure of QCD [4-6], which is known as the $U_{A}(1)$ anomaly. Here, $G$ denotes the $\mathrm{SU}(3)$ field strength tensor and $\tilde{G}$ its dual.

The connection between the $U_{A}(1)$-anomaly and the larger-than-expected mass of the $\eta^{\prime}$ meson is given by the famous Witten-Veneziano formula [7, 8], which is derived assuming the anomaly to be in place and in the large $N_{c}$ limit of QCD:

$$
\frac{f_{0}^{2}}{4 N_{f}}\left(M_{\eta}^{2}+M_{\eta^{\prime}}^{2}-2 M_{K}^{2}\right)=\chi_{\infty},
$$

Here, $\chi_{\infty}$ is the topological susceptibility in the pure YangMills (YM) gauge theory, $N_{f}$ the number of flavours, $f_{0}$ the flavour singlet decay constant and $M_{\eta}, M_{\eta^{\prime}}$ and $M_{K}$ are the $\eta$, the $\eta^{\prime}$ and the kaon masses, respectively. It relates nontrivial topological fluctuations of the gauge field in YM to the corresponding meson masses and singlet decay constant $f_{0}$ in QCD with $N_{f}$ active quark flavours. Using lattice gauge theory techniques we have the unique possibility to verify this relation.

In lattice QCD, the computation of $\eta$ and $\eta^{\prime}$ meson related quantities represents a challenge because of so-called fermionic disconnected diagrams. These are usually very

\footnotetext{
^e-mail: urbach@hiskp.uni-bonn.de
}

noisy and, therefore, the results available from lattice QCD were limited.

In the DFG funded project within the collaborative research centre CRC16 "Subnuclear Structre of Matter" we could increase the available lattice results in the flavoursinglet pseudoscalar sector significantly. This was possible on the one hand due to the lattice discretisation we are using: the Wilson twisted mass formulation at maximal twist is particularly well suited to study $\eta$ and $\eta^{\prime}$ mesons because of a powerful noise reduction method we developed in Ref. [9]. On the other hand we have applied and developed other methods which allow us to extract the quantities of interest with less uncertainty. Finally, we could perform this study employing a wide range of pion mass values, several strange quark mass values, several volumes and three values of the lattice spacing. Therefore, continuum and chiral extrapolations could be performed in a controlled way.

The results presented in the remaining sections represent an summary, update and extension of what was published in Refs. [10-15].

\section{Lattice Action}

The lattice QCD action for $N_{f}=2+1+1$ Wilson twisted mass fermions reads

$$
S=S_{g}+\bar{\chi}_{\ell} D_{\ell} \chi_{\ell}+\bar{\chi}_{h} D_{h} \chi_{h}
$$

For the gauge action $S_{g}$ we use the Iwasaki gauge action [16]. The twisted mass Dirac operator for the light - i.e. up/down quark - doublet reads [17]

$$
D_{\ell}=D_{W}+m_{0}+i \mu_{\ell} \gamma_{5} \tau^{3}
$$

and for the strange/charm doublet [18]

$$
D_{h}=D_{W}+m_{0}+i \mu_{\sigma} \gamma_{5} \tau^{1}+\mu_{\delta} \tau^{3},
$$

where $D_{W}$ is the Wilson Dirac operator and the $\tau^{i}$ are the Pauli matrices acting in flavour space. The value of $m_{0}$ was 
Table 1. compilation of values for the Sommer parameter $r_{0} / a$, the lattice spacing $a$ and $Z \equiv Z_{\mathrm{P}} / Z_{\mathrm{S}}$ determined with methods M1,M2 the three values of the lattice spacing. See Ref. [28] for details.

\begin{tabular}{lrrrr}
\hline$\beta$ & $Z(\mathbf{M 1})$ & $Z(\mathbf{M 2})$ & $a[\mathrm{fm}]$ & $r_{0} / a$ \\
\hline 1.90 & $0.699(13)$ & $0.651(06)$ & $0.0885(36)$ & $5.31(8)$ \\
1.95 & $0.697(07)$ & $0.666(04)$ & $0.0815(30)$ & $5.77(6)$ \\
2.10 & $0.740(05)$ & $0.727(03)$ & $0.0619(18)$ & $7.60(8)$ \\
\hline
\end{tabular}

tuned to its critical value $m_{\text {crit }}$ as discussed in Refs. [19, 20] in order to realise automatic $O(a)$ improvement at maximal twist [21]. The bare twisted masses $\mu_{\sigma}$ and $\mu_{\delta}$ are related to the bare strange and charm quark masses via the relation

$$
m_{c, s}=\mu_{\sigma} \pm Z \mu_{\delta}, \quad Z \equiv \frac{Z_{\mathrm{P}}}{Z_{\mathrm{S}}},
$$

where $Z$ denotes the ratio of non-singlet, pseudoscalar and scalar renormalisation constants $Z_{\mathrm{P}}$ and $Z_{\mathrm{S}}$. Quark fields in the twisted basis are denoted by $\chi_{\ell, h}$ and in the physical basis by $\psi_{\ell, h}$. They are related via the axial rotations

$$
\begin{aligned}
& \psi_{\ell}=e^{i \pi \gamma_{5} \tau^{3} / 4} \chi_{\ell}, \quad \bar{\psi}_{\ell}=\bar{\chi}_{\ell} e^{i \pi \gamma_{5} \tau^{3} / 4}, \\
& \psi_{h}=e^{i \pi \gamma_{5} \tau^{1} / 4} \chi_{h}, \quad \bar{\psi}_{h}=\bar{\chi}_{h} e^{i \pi \gamma_{5} \tau^{1} / 4} .
\end{aligned}
$$

With automatic $O(a)$ improvement being the biggest advantage of twisted mass lattice QCD (tmQCD) at maximal twist, the downside is that flavour (and parity) symmetry is broken at finite values of the lattice spacing. This was theoretically and numerically shown to affect mainly the mass value of the neutral pion [22-24]. For this reason we use masses of charged pions throughout this paper denoted as $M_{\mathrm{PS}}$. However, in the case of $N_{f}=2+1+1$ dynamical quarks, the flavour symmetry breaking implies the complication of mixing between strange and charm quarks. Note that the kaons are still degenerate, though.

We use gauge configurations as produced by the European Twisted Mass Collaboration (ETMC) with action described above [20, 25, 26]. The details of the configurations are described in Ref. [20] and we follow the notation adopted there: we use ensembles denoted with $A, B$ and $D$ with values of the lattice spacing $a_{A}=0.0885(36) \mathrm{fm}$, $a_{B}=0.0815(30) \mathrm{fm}$ and $a_{D}=0.0619(18) \mathrm{fm}$, corresponding to $\beta_{A}=1.90, \beta_{B}=1.95$ and $\beta_{D}=2.10$, respectively [26]. The physical volumes are - with only a few exceptions - larger than $3 \mathrm{fm}$.

Throughout this proceeding we will use the Sommer parameter $r_{0}$ [27] to investigate the scaling of our results. The actual numbers are taken from Ref. [28] and compiled in Table 1. In physical units we use $r_{0}=0.474(14) \mathrm{fm}$ taken from the same reference.

For fixing the light and strange quark masses to their physical values we will use the experimental values of $M_{\pi^{0}}=135 \mathrm{MeV}$ and $M_{\mathrm{K}^{0}}=498 \mathrm{MeV}$. We use the masses of the neutral mesons to reduce uncertainties from the fact that we do not include electromagnetic effects in our simulation. See also ref. [29] and references therein for a discussion.
For every $\beta$-value the bare values of $a \mu_{\sigma}$ and $a \mu_{\delta}$ were kept fixed, and only the value of the light twisted mass parameter $a \mu_{\ell}$ is varied. The kaon masses measured on these ensembles are close to the physical value with a deviation of up to $10 \%$ [20] in particular for the $A$ and $D$ ensembles. The D-meson mass values have a large uncertainty, but they are also close to physical [20].

For the $A$ lattice spacing we have two additional ensembles with re-tuned values of strange and charm-quark masses, denoted by $A 80.24 s$ and $A 100.24 s$, which reproduce the physical kaon mass value more accurately than the original $A 80.24$ and $A 100.24$ ensembles. We will use these ensembles to estimate the strange quark mass dependence of the $\eta$ mass and other quantities.

\section{Flavour-Singlet Pseudoscalar Mesons from Lattice QCD}

In order to compute masses of pseudoscalar flavour singlet mesons we have to include light, strange and charm contributions to build the appropriate correlation functions. In the light sector, one appropriate operator is given by [30]

$$
\frac{1}{\sqrt{2}}\left(-\bar{\chi}_{u} \chi_{u}+\bar{\chi}_{d} \chi_{d}\right) \equiv O_{\ell},
$$

in the twisted basis. With twisted mass fermions we have to work with doublets of quarks, hence, in the strange and charm sector the corresponding operators read

$$
\left(\begin{array}{c}
\bar{\chi}_{c} \\
\bar{\chi}_{s}
\end{array}\right)^{T} \frac{-\tau^{1} \pm i Z \gamma_{5} \tau^{3}}{2}\left(\begin{array}{l}
\chi_{c} \\
\chi_{s}
\end{array}\right) \equiv O_{c, s} .
$$

Note that the sum of pseudoscalar and scalar contributions appears with the ratio of renormalisation factors $Z$, which needs to be taken into account properly. The renormalisation factors for Eq. (8) are again the non-singlet $Z_{\mathrm{P}}$ and $Z_{\mathrm{S}}$. It is another advantage of the Wilson twisted mass formulation at maximal twist that only non-singlet renormalisation factors are required to obtain renormalised matrix elements. Defining $\vec{O}=\left(O_{l}, O_{c}, O_{s}\right)^{T}$, the correlation function matrix is given by

$$
C(t)=\sum_{\mathbf{x}}\langle\vec{O}(x) \otimes \vec{O}(0)\rangle
$$

Scalar and pseudoscalar contributions can be disentangled by changing the operator basis via an appropriate orthogonal matrix $\mathcal{R}$

$$
C_{\mathcal{R}}(t)=\langle\mathcal{R} \vec{O}(t) \otimes \mathcal{R} \vec{O}(0)\rangle=\mathcal{R} C(t) \mathcal{R}^{T},
$$

where

$$
C_{\mathcal{R}}=\left(\begin{array}{lll}
\eta_{O_{\ell} O_{\ell}} & \eta_{O_{\ell} \mathcal{S}_{h}} & \eta_{O_{\ell} \mathcal{P}_{h}} \\
\eta_{\mathcal{S}_{h} O_{\ell}} & \eta_{\mathcal{S}_{h} \mathcal{S}_{h}} & \eta_{\mathcal{S}_{h} \mathcal{P}_{h}} \\
\eta_{\mathcal{P}_{h} O_{\ell}} & \eta_{\mathcal{P}_{h} \mathcal{S}_{h}} & \eta_{\mathcal{P}_{h} \mathcal{P}_{h}}
\end{array}\right),
$$

is again a symmetric, real and positive definite correlation matrix with

$$
\begin{aligned}
\mathcal{P}_{h} & \equiv\left(\bar{\chi}_{c} i \gamma_{5} \chi_{c}-\bar{\chi}_{s} i \gamma_{5} \chi_{s}\right) / 2, \\
\mathcal{S}_{h} & \equiv Z^{-1}\left(\bar{\chi}_{s} \chi_{c}+\bar{\chi}_{c} \chi_{s}\right) / 2,
\end{aligned}
$$



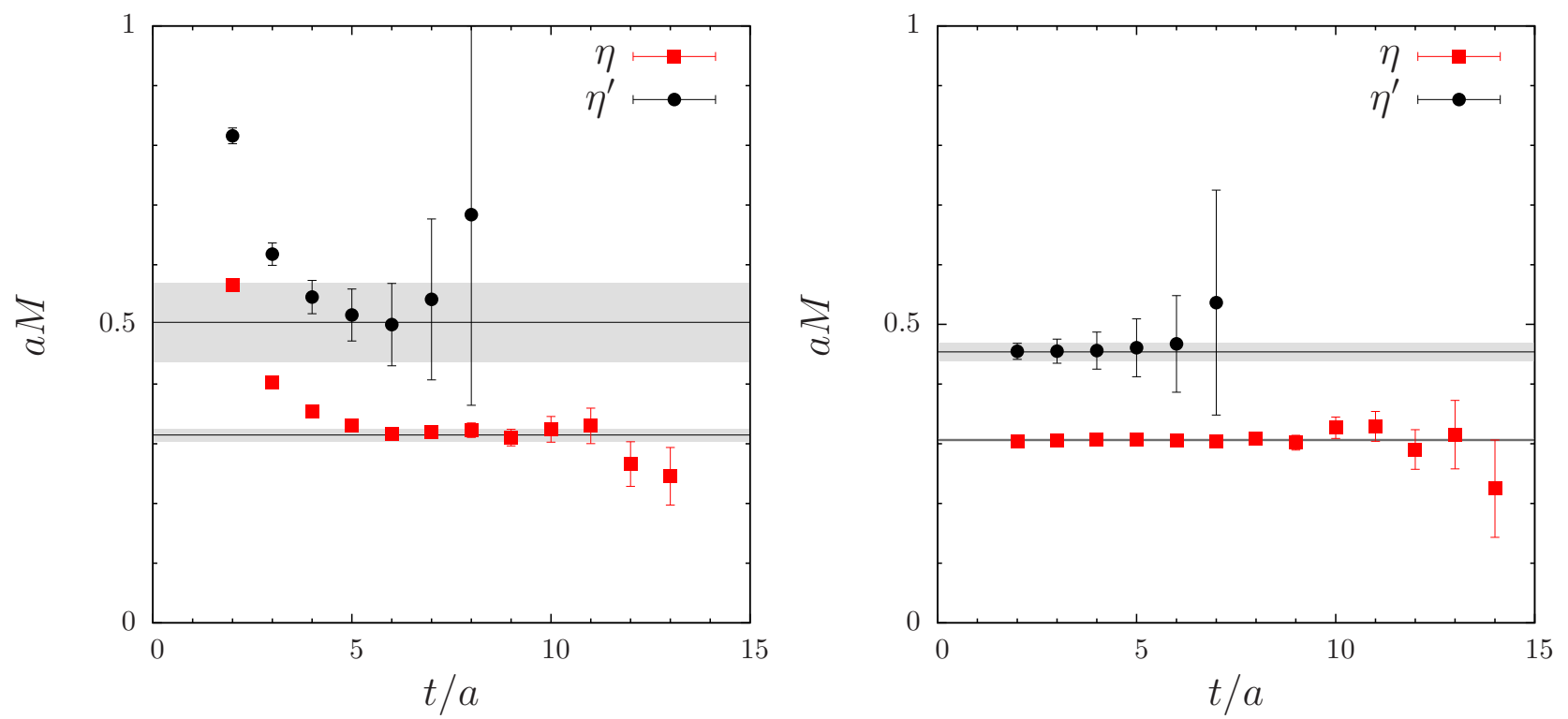

Figure 1. LefT: Effective masses in lattice units determined from solving the GEVP for a $6 \times 6$ matrix with $t_{0} / a=1$ for ensemble A100. Right: the same, but after removal of excited states in the connected contributions.

and $\eta_{X Y}$ denoting the corresponding correlation function. The rotation matrix $\mathcal{R}$ is given by

$$
\mathcal{R}=\left(\begin{array}{ccc}
1 & 0 & 0 \\
0 & -\frac{1}{\sqrt{2}} & -\frac{1}{\sqrt{2}} \\
0 & +\frac{1}{\sqrt{2}} & -\frac{1}{\sqrt{2}}
\end{array}\right) .
$$

Now we can drop the factor $Z^{-1}$, which appears only as a constant scaling factor for $\mathcal{S}_{h}$. We denote the corresponding correlation matrix by $\tilde{C}_{\mathcal{R}}$. Solving the generalised eigenvalue problem (GEVP) [31-33]

$$
\tilde{\mathcal{C}}_{\mathcal{R}}(t) \eta^{(n)}\left(t, t_{0}\right)=\lambda^{(n)}\left(t, t_{0}\right) \tilde{\mathcal{C}}_{\mathcal{R}}\left(t_{0}\right) \eta^{(n)}\left(t, t_{0}\right),
$$

and taking into account the periodic boundary conditions for a meson, we can determine the effective masses $M_{\mathrm{eff}}^{(n)}$ by solving

$$
\frac{\lambda^{(n)}\left(t, t_{0}\right)}{\lambda^{(n)}\left(t+1, t_{0}\right)}=\frac{e^{-M_{\mathrm{eff}}^{(n)} t}+e^{-M_{\mathrm{eff}}^{(n)}(T-t)}}{e^{-M_{\mathrm{eff}}^{(n)}(t+1)}+e^{-M_{\mathrm{eff}}^{(n)}(T-(t+1))}}
$$

for $M_{\mathrm{eff}}^{(n)}$, where $n$ counts the eigenvalues. The state with the lowest mass should correspond to the $\eta$ and the second state to the $\eta^{\prime}$ meson.

\subsection{Excited State Subtraction}

The result of solving the GEVP for a $6 \times 6$ matrix for ensemble A100 including fuzzed operators is shown as effective masses $M_{\text {eff }}$ in the left panel of Figure 1. It is visible that the ground state, the $\eta$ meson, can be extracted with good precision, while for the $\eta^{\prime}$ meson it is unclear that a plateau is reached before the signal is lost in noise.

However, there is a possibility to obtain a significant improvement for the extraction of $\eta^{\prime}$ mass (and further observables) using a powerful method to separate ground and excited states, which has first been proposed in Ref. [34] and that has already been successfully employed for the case of the $\eta_{2}$ for two dynamical quark flavours in Ref. [30], and we have first used it for the $N_{f}=2+1+1$ flavour case in Ref. [11].

The method is based on the assumption that the quark disconnected diagrams give a sizeable contribution only to the $\eta$ and $\eta^{\prime}$ state, but are negligible for any heavier state with the same quantum numbers. Considering the fluctuations of the topological charge which are expected to give a dominant contribution to the mass of the $\eta^{\prime}$, this assumption would be valid if these fluctuation mainly couple to the $\eta$ and $\eta^{\prime}$ states. Still, the validity of this assumption needs to be carefully checked from our data and may introduce systematic uncertainties.

Since the quark connected contributions exhibit a constant signal-to-noise ratio, it is in principle possible to determine the respective ground states at sufficiently large $t / a$ with high statistical accuracy and without any significant contamination from higher states. After fitting the respective ground states of the connected correlators, we can use it to subtract the excited state contributions such that the full connected correlators are replaced by correlators that contain only the ground state. Note that for sufficiently large $t / a$ this reproduces the original ground state by construction.

Now, if the aforementioned assumption holds, i.e. the disconnected diagrams are relevant only to the two lowest states $\eta, \eta^{\prime}$ one should obtain a plateau in the effective mass at very low values of $t / a$ after solving the GEVP. The result of the procedure is shown in the right panel of Figure 1. Indeed, one observes a plateau for both states starting basically at the lowest possible value of $t / a$. Furthermore, a comparison with the effective masses from the standard $6 \times 6$ matrix in the left panel of Figure 1 reveals that the plateau values agree very well within their respective errors. Most importantly, the data in the right panel allows for a much better accuracy in the determination of both masses as the point errors are much smaller at such 
low values of $t / a$. Therefore, we will use this method for all the results presented in this proceeding contribution.

However, we remark that in the twisted mass formulation with the non-degenerate doublet this procedure is in practice restricted to the connected correlation functions corresponding to physical light and strange quarks. This is due to the violation of flavour symmetry in the heavy sector of the twisted mass formulation, implying that the four connected contributions in the heavy sector will all yield the same ground state. This ground state corresponds to an artificial particle, i.e. a connected-only, neutral pionlike particle made out of strange quarks. Therefore, we will restrict ourselves in the following discussion to the analysis of a $2 \times 2$ correlation function matrix corresponding to (local) physical operators made of light and strange quarks.

We remark that this procedure modifies correlators only at small $t / a$ and per construction the limit of large Euclidean times is not modified. The method only serves to reduce statistical uncertainties by extending the plateaus to smaller values of $t / a$.

\subsection{Pollution by topological effects}

It is a well known problem, in particular for small lattice spacing values, that in lattice QCD simulations the topological charge is properly sampled [35]. This can lead in extreme cases to a freeze of the topological charge. We found in our ensembles that the topological charge has zero expectation value, however, the second moment of the charge distribution might not be sampled fully correctly. While being a finite volume effect, this can lead to time independent contributions to the flavour-singlet correlation function [36, 37]. As a consequence, the corresponding correlation functions do not decay to zero at large time separations.

Such constants present in correlation functions can be subtracted by fitting the appropriate functional form to the data [37]. However, we found that shifting the correlation functions - invented to cancel so-called thermal pollution in multi-hadron correlation functions [38] - subtracts the constant without the need of any additional input. Moreover, shifting decorrelates neighbouring time slices significantly, which gives an additional advantage.

We define the shifted correlation matrix as

$$
\tilde{C}_{\mathcal{R}}^{s}(t)=\tilde{C}_{\mathcal{R}}(t)-\tilde{C}_{\mathcal{R}}(t+\delta t)
$$

While all time independent terms are cancelled in $\tilde{C}_{\mathcal{R}}^{s}(t)$, every matrix element possesses now the following functional form

$$
\propto \sum_{n}\left[e^{-E_{n}(t+\delta t / 2)}-e^{-E_{n}(T-(t+\delta t / 2))}\right],
$$

with $T$ the time extent of the lattice. The shift $\delta t$ can be adjusted for optimal results.

We did not observe obvious cases in our data where the correlation functions themselves do not decay to zero. Only on a few ensembles with small volume and lattice spacings (e.g. D45) such a residual behaviour was observed for the resulting principal correlator of the $\eta^{\prime}$-state. However, in these cases the effect is of the size of a few standard deviations at most. Still, the shifting procedure turns out to systematically improve the signal for all and in particular for $\eta^{\prime}$ related quantities significantly. Final results of this procedure are currently in preparation [39], and we will only show $\eta$ and $\eta^{\prime}$ masses extracted with this procedure.

\subsection{Decay Constants and Mixing}

In general, decay constants are defined for any pseudoscalar meson $\mathrm{P}$ from axial vector matrix elements

$$
\left\langle 0\left|A_{\mu}^{a}\right| \mathrm{P}(p)\right\rangle=i f_{\mathrm{P}}^{a} p_{\mu},
$$

which leads to

$$
\left\langle 0\left|\partial^{\mu} A_{\mu}^{a}\right| \mathrm{P}(0)\right\rangle=f_{\mathrm{P}}^{a} M_{\mathrm{P}}^{2},
$$

for projection to zero momentum. Assuming that $\eta$ and $\eta^{\prime}$ are not flavour eigenstates, each of them exhibits a coupling to the singlet and octet axial vector current $A_{\mu}^{0}$ and $A_{\mu}^{8}$, respectively. Therefore, one ends up with four independent decay constants for the $\eta, \eta^{\prime}$-system, which are commonly parametrised in terms of two decay constants $f_{0}, f_{8}$ and two mixing angles $\theta_{0}, \theta_{8}$

$$
\begin{aligned}
\left(\begin{array}{ll}
f_{\eta}^{8} & f_{\eta}^{0} \\
f_{\eta^{\prime}}^{8} & f_{\eta^{\prime}}^{0}
\end{array}\right) & =\left(\begin{array}{cc}
f_{8} \cos \theta_{8} & -f_{0} \sin \theta_{0} \\
f_{8} \sin \theta_{8} & f_{0} \cos \theta_{0}
\end{array}\right) \\
& \equiv \Xi\left(\theta_{8}, \theta_{0}\right) \operatorname{diag}\left(f_{8}, f_{0}\right)
\end{aligned}
$$

The singlet decay constant $f_{0}$ needs renormalisation, determined by the anomalous dimension of the axial singlet current [40]. The dependence on the scale is $O\left(1 / N_{C}\right)$ and can, therefore, be dropped in the following discussion. For a detailed discussion see Refs. [41, 42].

We work in the quark flavour basis, with the axial vector currents $A_{\mu}^{0}$ and $A_{\mu}^{8}$ replaced by the combinations

$$
\begin{aligned}
& A_{\mu}^{l}=\frac{2}{\sqrt{3}} A_{\mu}^{0}+\sqrt{\frac{2}{3}} A_{\mu}^{8}=\frac{1}{\sqrt{2}}\left(\bar{u} \gamma_{\mu} \gamma_{5} u+\bar{d} \gamma_{\mu} \gamma_{5} d\right), \\
& A_{\mu}^{s}=\sqrt{\frac{2}{3}} A_{\mu}^{0}-\frac{2}{\sqrt{3}} A_{\mu}^{8}=\bar{s} \gamma_{\mu} \gamma_{5} s,
\end{aligned}
$$

in which the light quarks and the strange quark contributions are disentangled. In exact analogy to the singlet-octet basis this basis again allows for a parametrisation in terms of two decay constants and two mixing angles

$$
\left(\begin{array}{ll}
f_{\eta}^{l} & f_{\eta}^{s} \\
f_{\eta^{\prime}}^{l} & f_{\eta^{\prime}}^{s}
\end{array}\right)=\Xi\left(\phi_{l}, \phi_{s}\right) \operatorname{diag}\left(f_{l}, f_{s}\right),
$$

where the mixing matrix $\Xi$ has the same form as the one defined in Eq. (19). In this basis it is also expected that we have [41-45]

$$
\left|\frac{\phi_{l}-\phi_{s}}{\phi_{l}+\phi_{s}}\right| \ll 1,
$$



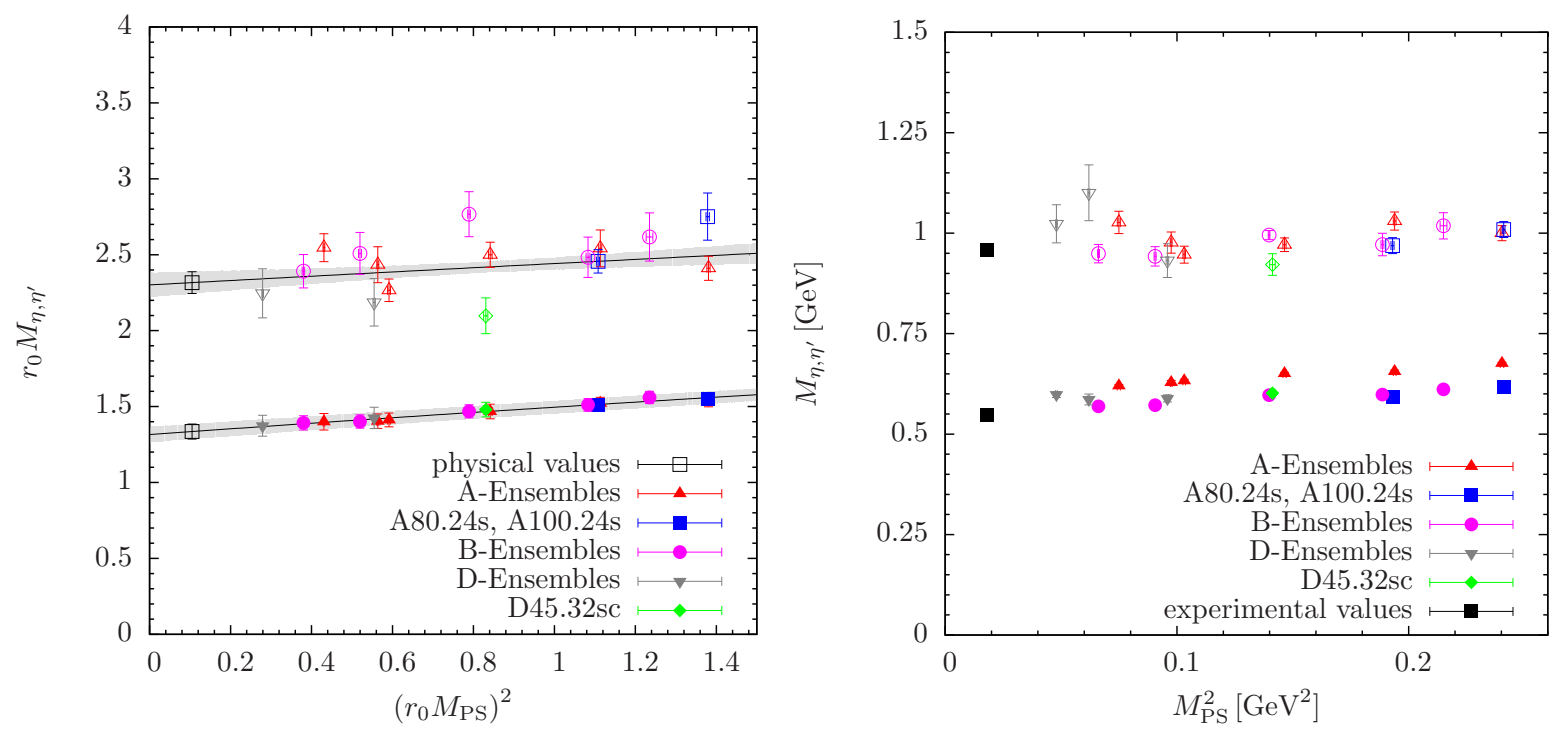

Figure 2. Left: Our results for $r_{0} \bar{M}_{\eta}$ (filled symbols) (corrected for the mismatch in $r_{0} M_{\mathrm{K}}$ ) and $r_{0} M_{\eta^{\prime}}$ (open symbols). The fitted curves are linear functions in $\left(r_{0} M_{\mathrm{PS}}\right)^{2}$ as discussed in the text. Right: $M_{\eta}$ (filled) and $M_{\eta^{\prime}}$ (open) in physical units (not corrected for the mismatch in $M_{\mathrm{K}}$ ) determined from the shifted correlation matrix as a function of $M_{\mathrm{PS}}^{2}$.

motivating a simplified mixing scheme in the quark flavour basis with only one angle $\phi$

$$
\left(\begin{array}{ll}
f_{\eta}^{l} & f_{\eta}^{s} \\
f_{\eta^{\prime}}^{l} & f_{\eta^{\prime}}^{s}
\end{array}\right)=\Xi(\phi) \operatorname{diag}\left(f_{l}, f_{s}\right)+O\left(\Lambda_{1}\right),
$$

where $\Xi(\phi) \equiv \Xi(\phi, \phi)$ and $\Lambda_{1}$ parametrises residual OZI violating terms. The mixing angle $\phi$ is related to the double ratio of amplitudes

$$
\tan ^{2}(\phi)=-\frac{f_{\eta^{\prime}}^{l} f_{\eta}^{s}}{f_{\eta}^{l} f_{\eta^{\prime}}^{s}}
$$

and hence independent of the ratio of renormalisation constants $Z$. Axial vector current matrix elements turn out to be difficult to measure in actual simulations due to noise. This is why it is most convenient to consider pseudoscalar currents in the quark flavour basis in analogy to Eqs. (20), (21).

$$
\begin{aligned}
P^{l} & =\frac{1}{\sqrt{2}}\left(\bar{u} \gamma_{5} u+\bar{d} \gamma_{5} d\right), \\
P^{s} & =\bar{s} i \gamma_{5} s,
\end{aligned}
$$

such that the matrix elements for pseudoscalar mesons $\mathrm{P}$ are given by

$$
h_{\mathrm{P}}^{i}=2 m_{i}\left\langle 0\left|P^{i}\right| \mathrm{P}\right\rangle,
$$

which are free from renormalisation. Making use of $\chi \mathrm{PT}$ and dropping sub-leading terms leads to the following expression [46]

$$
\left(\begin{array}{ll}
h_{\eta}^{l} & h_{\eta}^{s} \\
h_{\eta^{\prime}}^{l} & h_{\eta^{\prime}}^{s}
\end{array}\right)=\Xi(\phi) \operatorname{diag}\left(M_{\pi}^{2} f_{l},\left(2 M_{\mathrm{K}}^{2}-M_{\pi}^{2}\right) f_{s}\right) .
$$

This expression allows access to the decay constants $f_{s}$ and $f_{l}$ from pseudoscalar matrix elements under the assumption that $\chi \mathrm{PT}$ can be applied. In terms of pseudoscalar matrix elements the mixing angle $\phi$ is obtained as

$$
\tan ^{2}(\phi)=-\frac{h_{\eta^{\prime}}^{l} h_{\eta}^{s}}{h_{\eta}^{l} h_{\eta^{\prime}}^{s}},
$$

where actually quark masses and renormalisation constants drop out in the ratio. Expanding again to two angles, $\phi_{l}$ and $\phi_{s}$ are written as

$$
\tan \left(\phi_{l}\right)=\frac{h_{\eta^{\prime}}^{l}}{h_{\eta}^{l}}, \quad \tan \left(\phi_{s}\right)=-\frac{h_{\eta}^{s}}{h_{\eta^{\prime}}^{s}} .
$$

Finally, we remark that in order to compute the matrix elements $h_{P}^{i}$ in Wilson twisted mass lattice QCD the ratio of renormalisation constants $Z=Z_{P} / Z_{S}$ is required.

\section{Results}

\subsection{Masses}

We determine the masses $\tilde{C}_{\mathcal{R}}(t)$ using the excited state removal method described in the previous section. It turns out that in $a M_{\eta}$ we see a strange quark mass dependence that we can resolve well within our statistical errors. As the physical values of the strange quark masses vary by about $10 \%$ between the different lattice spacing values, we have to correct for this. This is described in detail in Ref. [10]. We use the ensembles A80 and A80s (A100 and A100s), which differ in the bare strange quark mass value, to estimate $D_{\eta}=d M_{\eta}^{2} / d M_{K}^{2}$. Next we correct all $a M_{\eta}$ values to correspond to a line of $M_{K}\left[M_{\mathrm{PS}}^{2}\right]$ values which reproduces the physical kaon mass at the physical pion mass point $M_{\mathrm{PS}}=M_{\pi^{0}}^{\mathrm{phys}}$. The such corrected values we denote with $\bar{M}_{\eta}$. Note that for this procedure we ignore any dependence of $D_{\eta}$ on the quark masses and the lattice spacing. For the $\eta^{\prime}$ mass we do not resolve quark mass or 


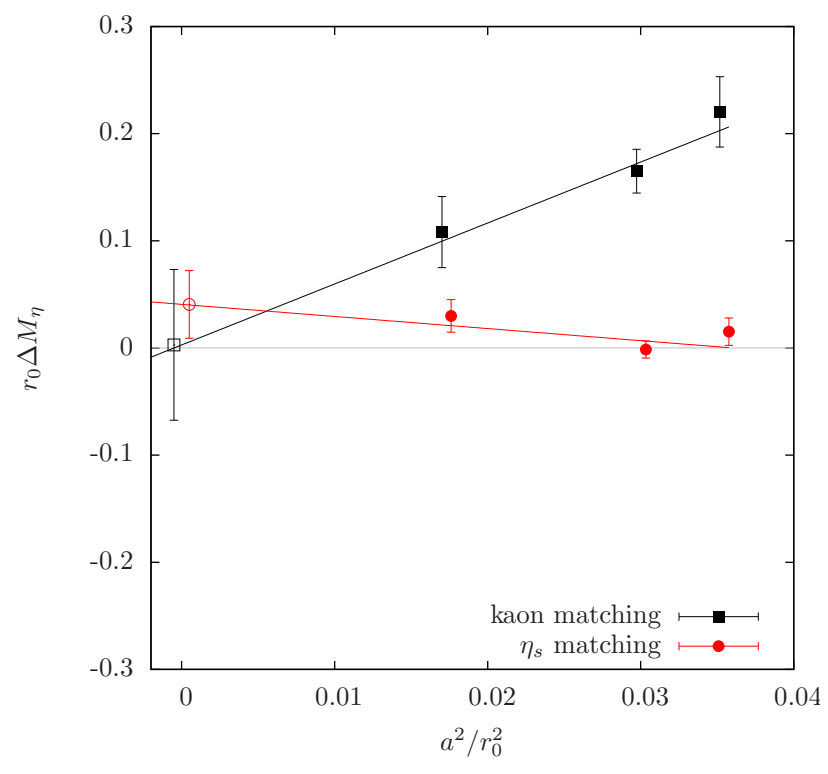

Figure 3. $r_{0} \Delta M_{\eta}$ as a function of $\left(a / r_{0}\right)^{2}$ for two matching procedures of OS valence strange quark to the unitary one [14].

lattice spacing dependence within our errors, so we do not attempt to correct for those.

The results for $\bar{M}_{\eta}$ and $M_{\eta^{\prime}}$ are summarised in Figure 2, where we show $r_{0} \bar{M}_{\eta}$ as filled and $M_{\eta^{\prime}}$ as open symbols, respectively, both as functions of $\left(r_{0} M_{\mathrm{PS}}\right)^{2}$. For both mesons the data fall on a single line within errors such that in both cases we model the data for $\left(r_{0} M\right)^{2}$ inspired by chiral perturbation theory as constant plus a linear term in $\left(r_{0} M_{\mathrm{PS}}\right)^{2}$. The corresponding best fit to the data and its error band is shown as lines with shaded bands. The error band for $M_{\eta}$ is mainly due to the error of $D_{\eta}$.

After extrapolating to the physical point and converting to physical units we obtain

$$
M_{\eta}\left(M_{\pi}\right)=556(8)_{\mathrm{stat}}(5)_{\mathrm{sys}} \mathrm{MeV},
$$

where the systematic error has been estimated from fitting to the data at each value of the lattice spacing separately. In addition, for the SU (2) chiral limit we find $r_{0} \stackrel{\circ}{\eta}^{\mathrm{SU}(2)}=$ $1.32(5)_{\text {stat }}(1)_{\text {sys }}$, which yields

$$
\stackrel{\circ}{M}_{\eta}^{\mathrm{SU}(2)}=548(9)_{\text {stat }}(6)_{\text {sys }} \mathrm{MeV} .
$$

We may extrapolate further quantities in order to check the validity of our correction procedure for mis-tuned values of the strange quark mass. First, we consider the GMO relation

$$
3 M_{\eta}^{2}=4 M_{\mathrm{K}}^{2}-M_{\pi}^{2},
$$

and build ratios left hand side over right hand side. This ratio is evaluated directly on our data. Next we perform an extrapolation in $\left(r_{0} M_{\mathrm{PS}}\right)^{2}$. However, it turns out that taking the uncorrected values of $M_{\eta}$ in the ratio the extrapolation misses the experimental value

$$
\left(3 M_{\eta}^{2} /\left(4 M_{\mathrm{K}}^{2}-M_{\pi}^{2}\right)\right)^{\exp }=0.926
$$

considering only the statistical error by more than $2 \sigma$, i.e. we obtain

$$
\left(3 M_{\eta}^{2} /\left(4 M_{\mathrm{K}}^{2}-M_{\pi}^{2}\right)\right)_{M_{\pi}}=0.965(15)_{\mathrm{stat}}(24)_{\mathrm{sys}} .
$$

This may be seen as a hint that the significantly increased statistical precision of the improved analysis strategy allows to resolve a residual strange quark mass dependence which is not cancelled in the ratio. This was not possible with the statistical accuracy we could obtain in Ref. [10]. Note that compared to the direct extrapolation the statistical precision is even further enhanced for the case of dimensionless ratios because the physical value of $r_{0}$ is only required for fixing the physical point, but not for the conversion to physical units. Considering only the $B$ ensembles for which the value of the strange quark mass is close to physical yields

$$
\left(3 M_{\eta}^{2} /\left(4 M_{\mathrm{K}}^{2}-M_{\pi}^{2}\right)\right)_{M_{\pi}}^{B}=0.928(26)_{\text {stat }},
$$

indicating that the systematic error is mainly caused by such a residual effect. Using the corrected values $\bar{M}_{\eta}$ and the corresponding values of the kaon mass to calculate the GMO ratio the extrapolation gives

$$
\left(3 M_{\eta}^{2} /\left(4 M_{\mathrm{K}}^{2}-M_{\pi}^{2}\right)\right)_{M_{\pi}}=0.960(19)_{\mathrm{stat}}(22)_{\mathrm{sys}},
$$

which is slightly closer to the experimental value. For the physical value of the $\eta$ mass we obtain

$$
M_{\eta}=558(6)_{\text {stat }}(7)_{\text {sys }} \mathrm{MeV} \text {, }
$$

in agreement with the result from the direct extrapolation and the experimental value.

A similar picture arises from the light quark mass extrapolation of the ratio $M_{\eta} / M_{K}$. Using the uncorrected values of $M_{\eta}$ which yields

$$
\left(M_{\eta} / M_{\mathrm{K}}\right)_{M_{\pi}}=1.117(8)_{\mathrm{stat}}(23)_{\mathrm{sys}},
$$

missing the experimental value

$$
\left(M_{\eta} / M_{\mathrm{K}}\right)^{\exp }=1.100
$$

again by roughly $2 \sigma$ if taking only the statistical error into account. Like for the case of the GMO ratio taking only the $B$ ensembles into account gives nice agreement with the experimental value, i.e.

$$
\left(M_{\eta} / M_{\mathrm{K}}\right)_{M_{\pi}}^{B}=1.093(14)_{\text {stat }},
$$

whereas $A$ and $D$ ensembles give significantly larger values. Therefore, we have also repeated the extrapolation using the corrected values $\bar{M}_{\eta}$ and the corresponding kaon masses. Within the statistical errors one obtains excellent agreement with experiment, i.e.

$$
\left(M_{\eta} / M_{\mathrm{K}}\right)_{M_{\pi}}=1.109(11)_{\mathrm{stat}}(09)_{\mathrm{sys}},
$$

which results in

$$
M_{\eta}=0.552(6)_{\text {stat }}(4)_{\text {sys }} \mathrm{MeV},
$$

compatible with the results from direct and GMO ratio extrapolations.

In order to obtain our final result for the physical mass of the $\eta$ meson we take the weighted average from the 

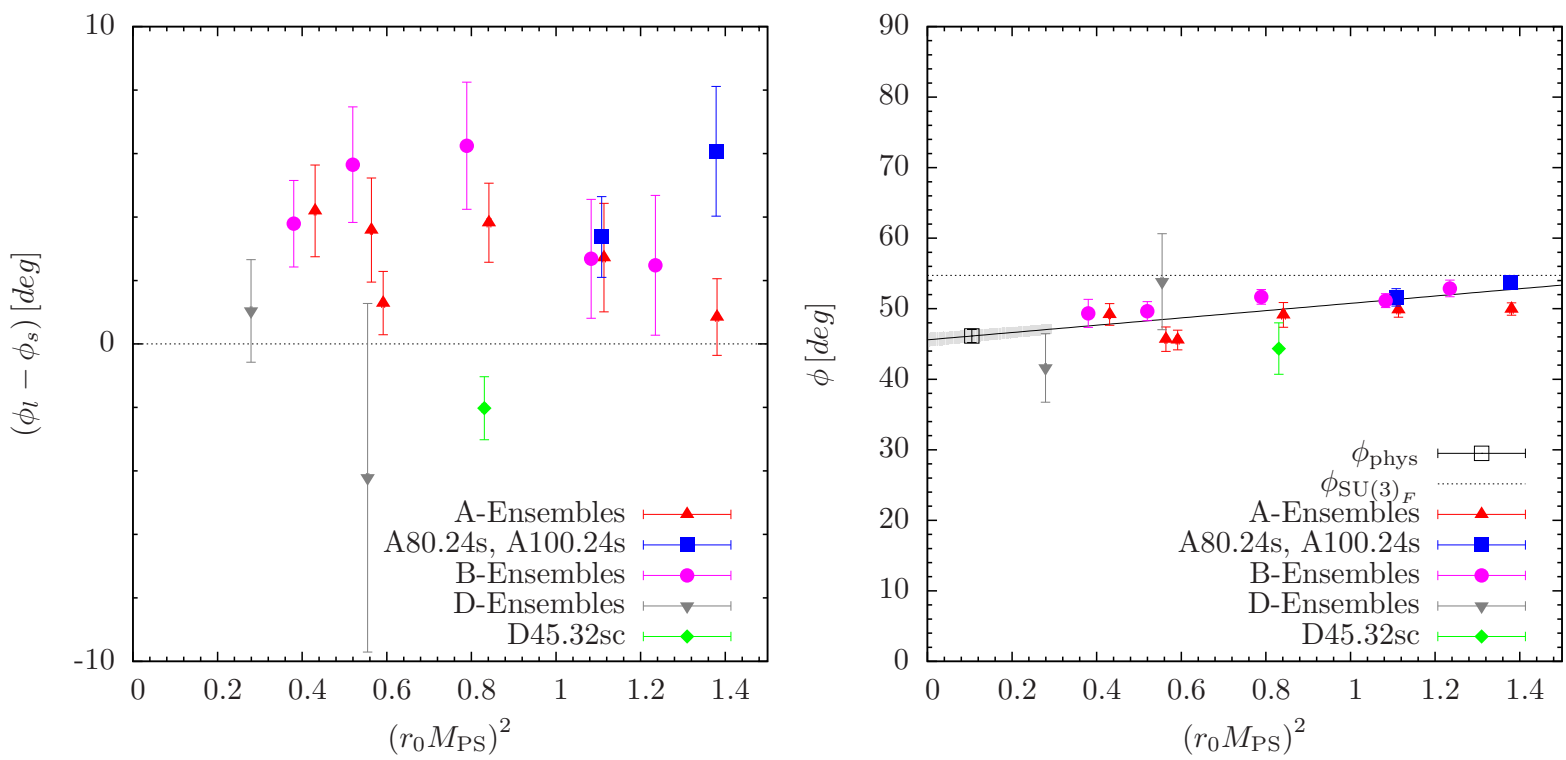

Figure 4. The difference $\phi_{l}-\phi_{s}$ (LEFT) and $\phi$ (RIGHT) in degrees as functions of $\left(r_{0} M_{\mathrm{PS}}\right)^{2}$. For $\phi$ we also show our best fit to the data and the extrapolation to the physical pion mass value. The dotted horizontal line represents the value $54.2^{\circ}$ one expects for $\phi$ in the $\mathrm{SU}(3)$ flavour symmetric case.

three previously discussed methods used for the extrapolation in the light quark mass. Accounting for any correlations, this yields

$$
M_{\eta}=555(6)_{\text {stat }}(5)_{\text {sys }} .
$$

which is in excellent agreement with experiment and exhibits substantially smaller errors compared to Ref. [10].

For the $\eta^{\prime}$ meson we obtain

$$
M_{\eta^{\prime}}=964(41)_{\mathrm{stat}}(34)_{\mathrm{sys}},
$$

from a linear extrapolation in $\left(r_{0} M_{\mathrm{PS}}\right)^{2}$ of all the (uncorrected) data. The corresponding fit is shown in the left panel of Figure 2. Again, the systematic error has been determined from fits to the data at single values of the lattice spacing. Within the larger errors this result is again in very good agreement with experiment, confirming that QCD indeed accounts for the significantly larger mass of the $\eta^{\prime}$ that is observed experimentally.

In the right panel of Figure 2 we show $M_{\eta, \eta^{\prime}}$ again as a function of $M_{\mathrm{PS}}^{2}$, but now $M_{\eta}$ and $M_{\eta^{\prime}}$ have been determined using the shifting procedure explained in section 3.2. The data is not corrected for the mismatch in the strange quark mass, and, therefore, the plot in the right panel is not directly comparable to the one in the left pane. But comparing it qualitatively to the left panel in the same figure shows that the shifting procedure greatly reduces the uncertainty in $M_{\eta^{\prime}}$, possibly to a level where strange quark and lattice spacing effects must be taken into account. A corresponding analysis is in progress [39].

\subsubsection{Mixed Action}

It is an interesting question whether or not quantities with a significant contribution of fermionic disconnected diagrams can be reliably determined in a so-called mixed ac- tion approach. In such an approach discretisation of valence and sea quark are different. Prime examples of quantities with large disconnected contribution are $\eta$ and $\eta^{\prime}$ meson masses.

We have investigated this question using the so-called Osterwalder-Seiler (OS) discretisation [47] for the valence strange quark combined with unitary up/down quarks on top of the Wilson twisted mass gauge configurations. The additional advantage of this mixed action approach is the avoided flavour mixing in the strange/charm sector. The conclusion of this investigation [14] is that indeed $\eta$ and $\eta^{\prime}$ masses can be determined reliably in this mixed action approach. But we observed that lattice artefacts strongly depend on the chosen matching quantity. As an example we show in Figure 3 the difference between the OS and the unitary $\eta$ meson mass

$$
\Delta M_{\eta}=M_{\eta}^{\mathrm{OS}}-M_{\eta}^{\text {unitary }}
$$

as a function of $\left(a / r_{0}\right)^{2}$. Results for two matching quantities are shown: one where the valence strange quark mass was fixed by matching the masses of the OS and the unitary $\eta_{s}$ meson. For the second the OS kaon was matched to the unitary for fixing the strange quark mass. As visible in Figure 3, kaon matching has significantly larger lattice artefacts, while they appear to be negligible for $\eta_{s}$ matching. Performing a continuum extrapolation of both matchings, however, gives identical results within error bars.

\subsection{Mixing Angles}

The mixing angles $\phi_{l}$ and $\phi_{s}$ can be extracted using Eq. (31) with a mixing model based on the pseudoscalar matrix elements $h_{P}^{q}$. In the left panel of Figure 4 we show $\phi_{l}-\phi_{s}$ in degrees as a function of $\left(r_{0} M_{\mathrm{PS}}\right)^{2}$. One observes that this quantity is consistent with zero within errors. Also an extrapolation to the physical point yields 

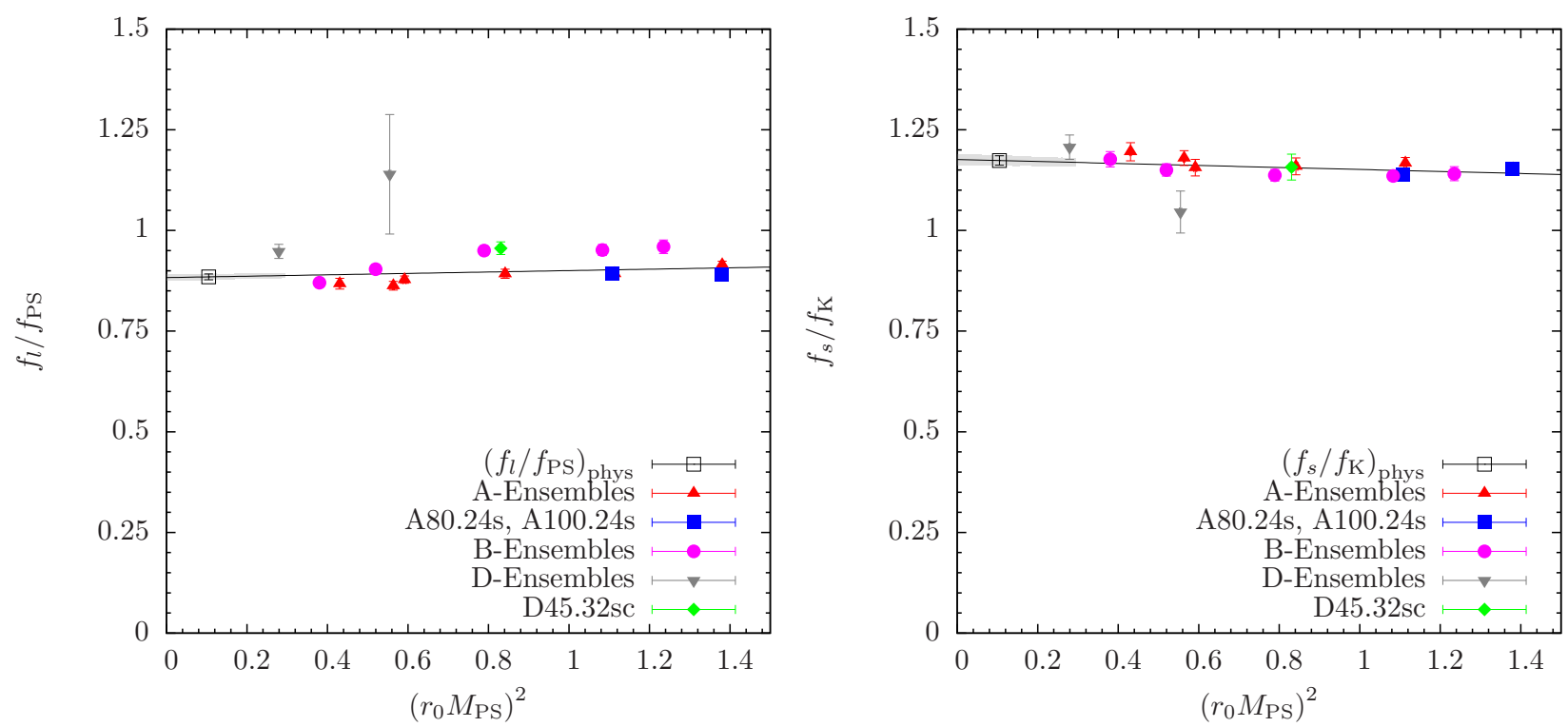

Figure 5. $f_{l} / f_{\mathrm{PS}}(\mathrm{LEFT})$ and $f_{l} / f_{K}$ (RIGHT) as functions of $\left(r_{0} M_{\mathrm{PS}}\right)^{2}$. The lines represent fits to our data with error band as explained in the text.

$3(1)_{\text {stat }}(3)_{\text {sys }}^{\circ}$, where the systematic error is estimated from the maximal difference compared to extrapolating the data sets for the three different lattice spacings separately.

In the right panel of Figure 4 we show the average angle $\phi$ (Eq. (30)) in degrees as a function of $\left(r_{0} M_{\mathrm{PS}}\right)^{2}$, with smaller statistical errors than $\phi_{l}$ and $\phi_{s}$ separately, because of correlation in the matrix elements. Our precision is not sufficient to resolve any residual lattice spacing or strange quark mass dependence. Hence, we extrapolate linearly in $\left(r_{0} M_{\mathrm{PS}}\right)^{2}$ and obtain

$$
\phi=46(1)_{\text {stat }}(3)_{\text {sys }}^{\circ},
$$

where the first error is statistical and the second systematic from fitting the three values of the lattice spacing separately.

Besides the mixing angle $\phi$, we consider the angles $\phi_{l}, \phi_{s}$ which are relevant to cross-check the assumptions entering our mixing scheme. Again, we have performed linear fits in $\left(r_{o} M_{\mathrm{PS}}\right)^{2}$ and obtain at the physical value of the pion mass

$$
\phi_{l}=48(1)_{\mathrm{stat}}(4)_{\mathrm{sys}}^{\circ}, \quad \phi_{s}=44(1)_{\mathrm{stat}}(3)_{\mathrm{sys}}^{\circ},
$$

where the systematic uncertainties have been determined in the same way as for the angle $\phi$ itself. The results are compatible within errors. Notably, for $\phi_{s}$ there is very good agreement for the results within each of the two set $(A 80.24, A 80.24 s)$ and $(A 100.24, A 100.24 s)$, indicating that the influence of the strange quark is smaller for this quantity and in general more of the data points lie within the error band of the linear fit.

\subsection{Decay Constants}

The application of the excited state removal method discussed in the previous section allows to extract the decay constants $f_{l}$ and $f_{s}$ to a rather high statistical precision by means of the pseudoscalar matrix elements $h_{P}^{i}$ and using Eq. (29). Of course, one needs to keep in mind that this is based on the assumption that the underlying chiral perturbation theory analysis is valid. Currently, we cannot estimate a corresponding systematic uncertainty.

In Figure 5 we show $f_{l} / f_{\mathrm{PS}}$ and $f_{s} / f_{K}$ as functions of $\left(r_{0} M_{\mathrm{PS}}\right)^{2}$ in the left and right panel, respectively. We have chosen to plot these ratios because it appears that most of the quark mass and lattice spacing dependence cancels. A linear extrapolation of $f_{l} / f_{\mathrm{PS}}$ in $\left(r_{0} M_{\mathrm{PS}}\right)^{2}$ to the physical point results in

$$
f_{l} / f_{\pi}=0.884(7)_{\text {stat }}(63)_{\text {sys }} .
$$

However, from the plot it appears that there is still a rather sizeable dependence on the lattice spacing present while the strange quark mass dependence seems to cancel in the ratio as all $A$-ensembles fall on one single curve. In fact, the systematic error estimated from fitting the data at each value of the lattice spacing separately is one order of magnitude larger than the statistical error and there is clear trend towards larger values of $f_{l} / f_{\mathrm{PS}}$ extrapolated to the physical pion mass for decreasing values of the lattice spacing. Therefore, we additionally quote the result of a linear fit restricted to the data at the finest lattice spacing, which yields

$$
\left(f_{l} / f_{\pi}\right)^{D}=0.947(23)_{\text {stat }} .
$$

For the ratio $f_{s} / f_{K}$ most of the strange quark mass dependence is cancelled and the data seem almost perfectly linear in the light quark mass, exhibiting only a moderate slope. Moreover, for this case there are no discernible scaling artefacts within errors and the data are well described by a linear fit which gives

$$
f_{s} / f_{K}=1.176(12)_{\text {stat }}(28)_{\text {sys }}
$$

at the physical value of the pion mass. Clearly, the systematic error is significantly smaller than the one obtained 


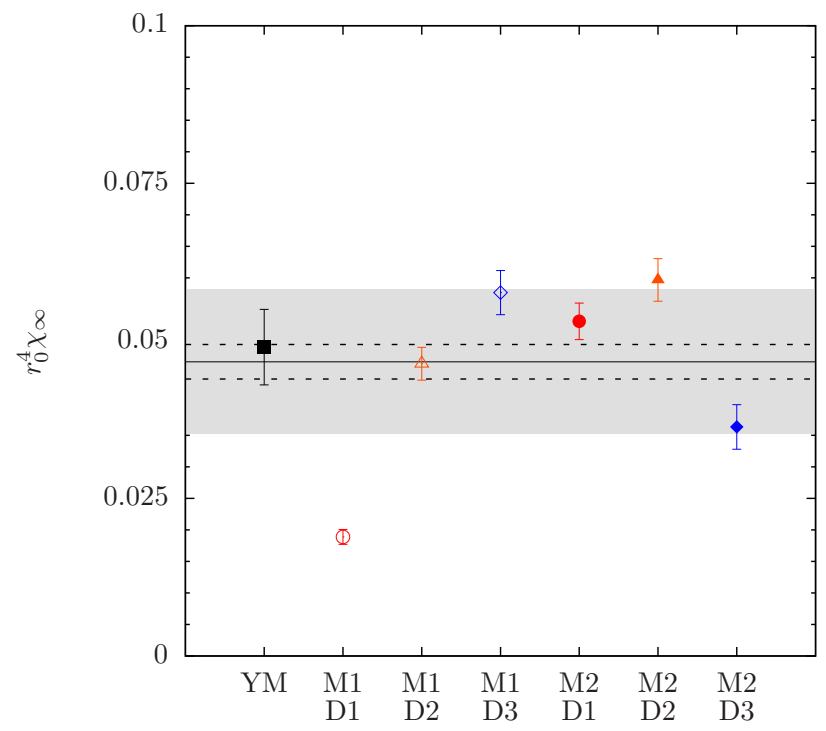

Figure 6. A comparison of the YM topological susceptibility $\chi_{\infty}$ to the right hand side of the Witten-Veneziano formula. The latter is determined using two different renormalisation procedures (M1, M2) and three different ways to estimate $f_{0}(\mathbf{D 1}, \mathbf{D 2}, \mathbf{D 3})$.

for the physical value of $f_{l} / f_{\pi}$, confirming the smallness of any residual lattice artefacts or strange quark mass dependence for $f_{s} / f_{K}$.

Finally we have to remark that finite volume effects might play an important role for the decay constants, but they hopefully cancel in the ratios we used.

\subsection{Witten-Veneziano Formula}

Now we have all ingredients at hand to investigate the Witten-Veneziano formula in more detail. As we have shown in Ref. [15], one can relate the decay constants $f_{\ell}$ and $f_{s}$ to the singlet decay constant $f_{0}$ and the kaon and pion decay constants $f_{K}$ and $f_{\pi}$ using $\chi \mathrm{PT}$ again

$$
\begin{aligned}
& f_{0}^{2}=-7 / 6 f_{\pi}^{2}+2 / 3 f_{K}^{2}+3 / 2 f_{\ell}^{2}, \\
& f_{0}^{2}=+1 / 3 f_{\pi}^{2}-4 / 3 f_{K}^{2}+f_{\ell}^{2}+f_{s}^{2}, \\
& f_{0}^{2}=+3 f_{s}^{2}-\frac{8}{3}\left(2 f_{K}^{2}-f_{\pi}^{2}\right) .
\end{aligned}
$$

respectively. We denote these three formulae for $f_{0}$ with D1, D2, D3. $f_{0}$ is not uniquely determined because of higher order terms that have been dropped, see Ref. [15] for details. These three equations together with the methods M1 and M2 to determine the renormalisation constants [28], lead to six determinations of the left hand side of Eq. (1), which are compared to the YM susceptibility in Figure 6. We have determined the susceptibility $\chi_{\infty}$ in a dedicated simulation of the YM theory with three values of the lattice spacing applying the so-called spectral projector method [48].

This comparison is only meaningful up to higher order contributions in $1 / N_{c}$. Therefore, one cannot expect exact agreement. However, qualitatively the observed agreement is very good.

\subsection{Decay Widths $\Gamma_{P \rightarrow \gamma \gamma}$}

The decay constants $f_{l}$ and $f_{s}$ are important low energy constants. However, they can also be used to estimate phenomenologically interesting quantities, most prominently the decay widths of $\eta, \eta^{\prime} \rightarrow \gamma \gamma$. To the same order in the effective theory we worked until here, one can relate the decays widths with the mixing parameters in the quark flavour basis as follows [50, 51] (see Refs. [41, 42] for how to include these quantities into the effective field theory framework)

$$
\begin{aligned}
\Gamma_{\eta \rightarrow \gamma \gamma} & =\frac{\alpha^{2}}{288 \pi^{3}} M_{\eta}^{3}\left[\frac{5}{f_{l}} \cos \phi-\frac{\sqrt{2}}{f_{s}} \sin \phi\right]^{2}, \\
\Gamma_{\eta^{\prime} \rightarrow \gamma \gamma} & =\frac{\alpha^{2}}{288 \pi^{3}} M_{\eta^{\prime}}^{3}\left[\frac{5}{f_{l}} \sin \phi+\frac{\sqrt{2}}{f_{s}} \cos \phi\right]^{2},
\end{aligned}
$$

where again OZI suppressed terms have been dropped and our normalisation is that $f_{\pi}=130.7 \mathrm{MeV}$. Using our lattice data for $M_{\eta, \eta^{\prime}}, \phi, f_{l}$ and $f_{s}$, we have computed the decay widths and show them in units of the Sommer parameter as a function of $\left(r_{0} M_{\mathrm{PS}}\right)^{2}$ in Figure 7 . We also include the PDG value for convenience [49]. For $\Gamma_{\eta^{\prime} \rightarrow \gamma \gamma}$ we observe a flat dependence of the light quark mass and agreement between our data and the PDG value within our errors. However, the data show a tendency for lower values with decreasing values of the lattice spacing.

The situation is not so clear for $\Gamma_{\eta \rightarrow \gamma \gamma}$, where the lattice data is of the right magnitude compared to the PDG value. But there are clearly strange quark mass and light quark mass effects visible that we cannot control at the moment. In addition there might be lattice artefacts.

Two remarks are in order: first of all the mass dependence of the widths has not been computed in effective field theory. Hence, we have no rigorous means to extrapolate our data to the physical point. Eqs. (46) are strictly speaking only valid in the chiral limit. Second, the PDG value for $\Gamma_{\eta \rightarrow \gamma \gamma}$ does not include Primakoff experiments, which give a significantly smaller value ${ }^{1}$.

Related quantities are the pseudoscalar transition form factors $F_{\eta \gamma \gamma^{*}}$ and $F_{\eta^{\prime} \gamma \gamma^{*}}$ for large momentum transfer $q^{2}$, which can be expressed as [52]

$$
\begin{aligned}
\lim _{q^{2} \rightarrow \infty} q^{2} F_{\eta \gamma \gamma^{*}}\left(q^{2}\right) & =\frac{10}{3 \sqrt{2}} f_{l} \cos \phi-\frac{2}{3} f_{s} \sin \phi \\
& =100(5)_{\text {stat }}(25)_{\text {sys }} \mathrm{MeV} \\
\lim _{q^{2} \rightarrow \infty} q^{2} F_{\eta^{\prime} \gamma \gamma^{*}}\left(q^{2}\right) & =\frac{10}{3 \sqrt{2}} f_{l} \sin \phi+\frac{2}{3} f_{s} \cos \phi \\
& =277(3)_{\text {stat }}(20)_{\text {sys }} \mathrm{MeV}
\end{aligned}
$$

These results have to be understood to be very preliminary. They are obtained by using the values of $f_{l} / f_{\pi}$ and $f_{s} / f_{K}$ in Eqs. (41) and (42), respectively, together with the physical values of the pion and kaon decay constants $f_{\pi}=130.42 \mathrm{MeV}$ and $f_{K}=156.2 \mathrm{MeV}$ [53]. The value for $\phi$ has been taken from Eq. (38). The systematic uncertainty is again calculated from the maximal difference to results from the separate lattice spacing values.

\footnotetext{
${ }^{1}$ We thank P. Masjuan for drawing our attention to this fact.
} 

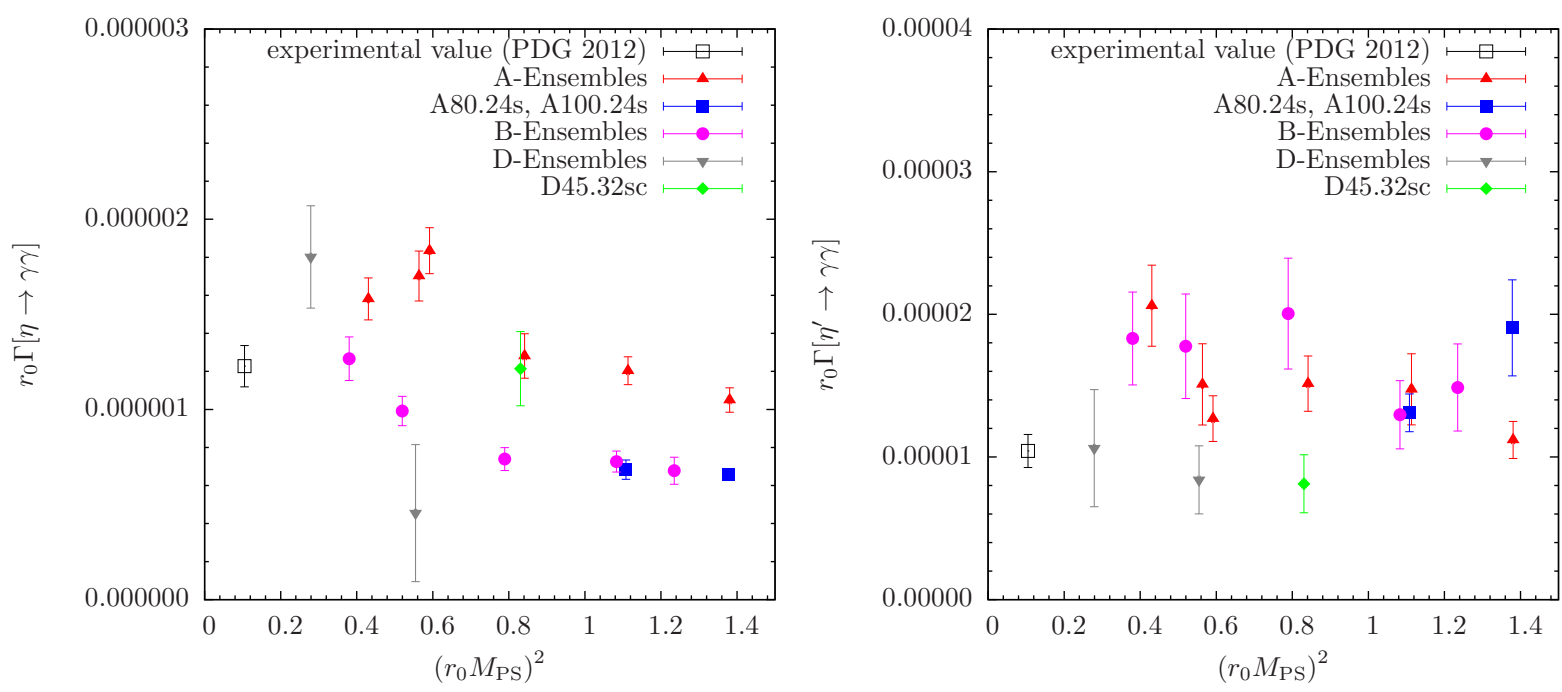

Figure 7. We show the decays widths $\Gamma_{\eta \rightarrow \gamma \gamma}$ (LEFT) and $\Gamma_{\eta^{\prime} \rightarrow \gamma \gamma}$ (RIGHT) in units of $r_{0}$ as functions of $\left(r_{0} M_{\mathrm{PS}}\right)^{2}$. In addition we show the corresponding estimate from the PDG [49].

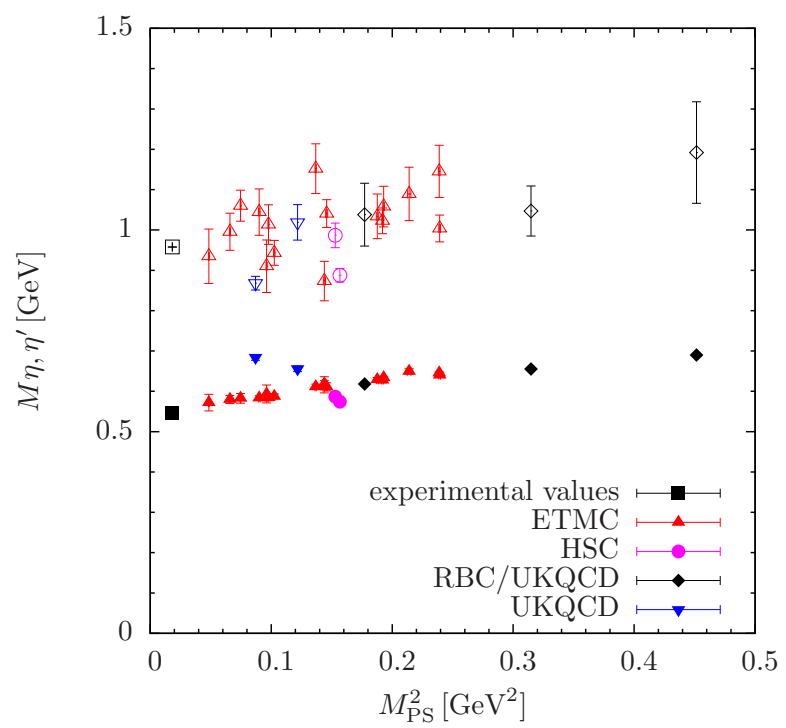

Figure 8. Results for $M_{\eta}$ and $M_{\eta^{\prime}}$ in physical units as a function of $M_{\mathrm{PS}}^{2}$ compared to results from other lattice QCD computations (HSC: Ref. [54], RBC/UKQCD: Ref. [55] and UKQCD Ref. [56]). Open symbols represent the $\eta^{\prime}$ and filled symbols the $\eta$ mass, respectively.

\section{Summary}

In this proceeding we have summarised the results obtained for properties of flavour-singlet pseudoscalar mesons from lattice QCD as part of the collaborative research centre CRC16.

In Figure 8 we show a compilation of our results for $\eta$ and $\eta^{\prime}$ masses together with results available in the literature for $N_{f}=2+1$ flavour lattice QCD. For $M_{\eta}$ we show the values corrected for the mismatch in $M_{\mathrm{K}}$. We remark that in Ref. [57] $\eta$ and $\eta^{\prime}$ meson masses have been computed using $N_{f}=2+1$ flavours of overlap quarks at one value of the lattice spacing and large values of the pion mass, however, in this reference not enough details are given to be included in our comparison Figure 8 . The results in Ref. [55] have been obtained using $N_{f}=2+1$ flavours of domain wall fermions and again for a single value of the lattice spacing $a \approx 0.1 \mathrm{fm}$ but for three values of the pion mass in a range from $\sim 400 \mathrm{MeV}$ to $\sim 700 \mathrm{MeV}$. The corresponding data points in Figure 2 are labelled "RBC/UK QCD". Another single data point is added from Ref. [54] by the Hadron Spectrum Collaboration (HSC) for which Wilson fermions have been employed. Again, it was not possible to include more recent results by the HSC [58] due to the lack of explicit numerical values in this reference for the relevant masses. Finally, in Ref. [56] data from staggered fermions are presented for two different values of the lattice spacing with each of them also at a different value of the pion mass. In Figure 8 the corresponding data points are labelled "UKQCD". The figure suggests an overall agreement between all collaborations.

In Table 2 we compare our results for various quantities to experimental results from the PDG [53] and to phenomenological results from the so-called FKS-scheme taken from Ref. [46] (see also Ref. [52]). We observe mostly quite good agreement with the exception of $f_{\ell} / f_{\pi}$. This exception is likely a systematic uncertainty coming from the fact that in this proceeding we did not yet include lattice artefacts in the analysis of $f_{\ell} / f_{\pi}$. A corresponding analysis is ongoing [39]. Note that in general the spread in the phenomenological determinations is substantial.

As discussed in Ref. [52], this determination of mixing parameters can be used to better estimate the hadronic light-by-light contribution to the anomalous magnetic moment of the muon. Of course, eventually a computation of the corresponding transition form factors is desired as recently performed for the neutral pion in Refs. [59, 60].

Finally, we have investigated the Witten-Veneziano formula by computing all for the formula relevant quantities non-perturbatively. The results indicate clearly that the axial anomaly in QCD is able to explain the large mass of the $\eta^{\prime}$ meson (see also Ref. [61]). 
Table 2. Summary of results. The literature values are taken from Ref. [53] for $M_{\eta}$ and $M_{\eta^{\prime}}$ and from Ref. [46] from the so-called FKS scheme for the others.

\begin{tabular}{lrr}
\hline & this work & literature \\
\hline$M_{\eta}[\mathrm{MeV}]$ & $555(6)_{\text {stat }}(5)_{\text {sys }}$ & $547.86(2)$ \\
$M_{\eta^{\prime}}[\mathrm{MeV}]$ & $964(41)_{\text {stat }}(34)_{\text {sys }}$ & $957.78(6)$ \\
$f_{\ell} / f_{\pi}$ & $0.884(7)_{\text {stat }}(63)_{\text {sys }}$ & $1.07(2)$ \\
$f_{s} / f_{K}$ & $1.176(12)_{\text {stat }}(28)_{\text {sys }}$ & $1.11(5)$ \\
$\phi$ & $46(1)_{\text {stat }}(3)_{\text {sys }}^{\circ}$ & $39(1)^{\circ}$ \\
\hline
\end{tabular}

The author wishes to thank K. Ottnad and C. Michael for their essential contributions to this project. Thanks also to Falk Zimmermann for his work on the mixed action approach. The work reported here would not have been possible without the support from the Deutsche Forschungsgemeinschaft within the SFB/TR16. We thank the members of ETMC for the most enjoyable collaboration. The computer time for this project was made available to us by the John von Neumann-Institute for Computing (NIC) on the JUDGE, Jugene and Juqueen systems in Jülich. Support by the Bonn-Cologne Graduate School (BCGS) of Physics and Astronomie is acknowledged. The open source software packages tmLQCD [62-64], Lemon [65] and R [66] have been used.

\section{References}

[1] S. Weinberg, Phys.Rev. D11, 3583 (1975)

[2] S.L. Adler, Phys. Rev. 177, 2426 (1969)

[3] J.S. Bell, R. Jackiw, Nuovo Cim. A60, 47 (1969)

[4] J.B. Kogut, L. Susskind, Phys. Rev. D11, 3594 (1975)

[5] A.A. Belavin, A.M. Polyakov, A.S. Schwartz, Yu.S. Tyupkin, Phys. Lett. B59, 85 (1975)

[6] G. 't Hooft, Phys. Rev. Lett. 37, 8 (1976)

[7] E. Witten, Nucl.Phys. B156, 269 (1979)

[8] G. Veneziano, Nucl.Phys. B159, 213 (1979)

[9] C. Michael, C. Urbach (ETM), PoS LAT2007, 122 (2007), 0709.4564

[10] K. Ottnad, C. Michael, S. Reker, C. Urbach, C. Michael, S. Reker, C. Urbach (ETM), JHEP 11, 048 (2012), 1206.6719

[11] C. Michael, K. Ottnad, C. Urbach (ETM), Phys. Rev. Lett. 111, 181602 (2013), 1310. 1207

[12] C. Michael, K. Ottnad, C. Urbach (ETM), PoS LATTICE2013, 253 (2014), 1311.5490

[13] K. Ottnad, Ph.D. thesis, Bonn U. (2013), hss.ulb. uni-bonn. de/2014/3506/3506.htm

[14] K. Ottnad, C. Urbach, F. Zimmermann (OTM), Nucl. Phys. B896, 470 (2015), 1501.02645

[15] K. Cichy, E. Garcia-Ramos, K. Jansen, K. Ottnad, C. Urbach (ETM), JHEP 09, 020 (2015), 1504.07954

[16] Y. Iwasaki, Nucl. Phys. B258, 141 (1985)

[17] R. Frezzotti, P.A. Grassi, S. Sint, P. Weisz (ALPHA), JHEP 08, 058 (2001), hep-lat/0101001
[18] R. Frezzotti, G.C. Rossi, Nucl. Phys. Proc. Suppl. 128, 193 (2004), hep-lat/0311008

[19] T. Chiarappa et al., Eur. Phys. J. C50, 373 (2007), hep-lat/0606011

[20] R. Baron et al. (ETM), JHEP 06, 111 (2010), 1004.5284

[21] R. Frezzotti, G.C. Rossi, JHEP 08, 007 (2004), hep-lat/0306014

[22] R. Baron et al. (ETM), JHEP 1008, 097 (2010), Q911.5061

[23] C. Urbach (ETM), PoS LAT2007, 022 (2007), Q710.1517

[24] P. Dimopoulos, R. Frezzotti, C. Michael, G. Rossi, C. Urbach, Phys.Rev. D81, 034509 (2010), Q908.0451

[25] R. Baron et al. (ETM), Comput.Phys.Commun. 182, 299 (2011), 1005.2042

[26] R. Baron et al., PoS LATTICE2010, 123 (2010), 1101.0518

[27] R. Sommer, Nucl.Phys. B411, 839 (1994), hep-lat/9310022

[28] N. Carrasco et al. (ETM), Nucl. Phys. B887, 19 (2014), 1403.4504

[29] G. Colangelo, S. Durr, A. Juttner, L. Lellouch, H. Leutwyler et al., Eur.Phys.J. C71, 1695 (2011), 1011.4408

[30] K. Jansen, C. Michael, C. Urbach (ETM), Eur.Phys.J. C58, 261 (2008), 0804 3871

[31] C. Michael, I. Teasdale, Nucl.Phys. B215, 433 (1983)

[32] M. Lüscher, U. Wolff, Nucl.Phys. B339, 222 (1990)

[33] B. Blossier, M. Della Morte, G. von Hippel, T. Mendes, R. Sommer, JHEP 0904, 094 (2009), Q902.1265

[34] H. Neff, N. Eicker, T. Lippert, J.W. Negele, K. Schilling, Phys.Rev. D64, 114509 (2001), hep-lat/0106016

[35] S. Schaefer, R. Sommer, F. Virotta (ALPHA), Nucl. Phys. B845, 93 (2011), 1009. 5228

[36] S. Aoki, H. Fukaya, S. Hashimoto, T. Onogi, Phys. Rev. D76, 054508 (2007), 0707.0396

[37] G.S. Bali, S. Collins, S. D?rr, I. Kanamori, Phys. Rev. D91, 014503 (2015), 1406. 5449

[38] X. Feng, K. Jansen, D.B. Renner, Phys.Lett. B684, 268 (2010), 0909. 3255

[39] K. Ottnad, C. Urbach (2016), in preparation

[40] J. Kodaira, Nucl.Phys. B165, 129 (1980)

[41] R. Kaiser, H. Leutwyler (1998), hep-ph/9806336

[42] R. Kaiser, H. Leutwyler, Eur.Phys.J. C17, 623 (2000), hep-ph/0007101

[43] J. Schechter, A. Subbaraman, H. Weigel, Phys.Rev. D48, 339 (1993), hep-ph/9211239

[44] T. Feldmann, P. Kroll, B. Stech, Phys.Lett. B449, 339 (1999), hep-ph/9812269

[45] T. Feldmann, P. Kroll, B. Stech, Phys.Rev. D58, 114006 (1998), hep-ph/9802409 
[46] T. Feldmann, Int.J.Mod.Phys. A15, 159 (2000), hep-ph/9907491

[47] R. Frezzotti, G.C. Rossi, JHEP 10, 070 (2004), hep-lat/0407002

[48] L. Giusti, M. Luscher, JHEP 03, 013 (2009), 0812.3638

[49] J. Beringer et al. (Particle Data Group), Phys.Rev. D86, 010001 (2012)

[50] T. Feldmann, P. Kroll, Eur.Phys.J. C5, 327 (1998), hep-ph/9711231

[51] T. Feldmann, P. Kroll, Phys.Scripta T99, 13 (2002), hep-ph/0201044

[52] R. Escribano, P. Masjuan, P. Sanchez-Puertas (2013), 1307.2061

[53] K.A. Olive et al. (Particle Data Group), Chin. Phys. C38, 090001 (2014)

[54] J.J. Dudek, R.G. Edwards, B. Joo, M.J. Peardon, D.G. Richards et al., Phys.Rev. D83, 111502 (2011), 1102.4299

[55] N. Christ, C. Dawson, T. Izubuchi, C. Jung, Q. Liu et al., Phys.Rev.Lett. 105, 241601 (2010), 1002.2999

[56] E.B. Gregory, A.C. Irving, C.M. Richards, C. McNeile (UKQCD), Phys.Rev. D86, 014504 (2012), 1112.4384
[57] T. Kaneko et al. (TWQCD/JLQCD), PoS LAT2009, 107 (2009), 0910. 4648

[58] J.J. Dudek, R.G. Edwards, P. Guo, C.E. Thomas (2013), 1309.2608

[59] X. Feng, S. Aoki, H. Fukaya, S. Hashimoto, T. Kaneko et al., Phys.Rev.Lett. 109, 182001 (2012), 1206.1375

[60] A. Gérardin, H.B. Meyer, A. Nyffeler (2016), 1607.08174

[61] M. Cè, M.G. Vera, L. Giusti, S. Schaefer (2016), 1607.05939

[62] K. Jansen, C. Urbach, Comput.Phys.Commun. 180, 2717 (2009), 0905 . 3331

[63] A. Abdel-Rehim, F. Burger, A. Deuzeman, K. Jansen, B. Kostrzewa, L. Scorzato, C. Urbach, PoS LATTICE2013, 414 (2014), 1311.5495

[64] A. Deuzeman, K. Jansen, B. Kostrzewa, C. Urbach, PoS LATTICE2013, 416 (2014), 1311 . 4521

[65] A. Deuzeman, S. Reker, C. Urbach, Comput.Phys.Commun. 183, 1321 (2012), 1106. 4177

[66] R Development Core Team, $R$ : A language and environment for statistical computing, $\mathrm{R}$ Foundation for Statistical Computing, Vienna, Austria (2005), ISBN 3-900051-07-0, http://www.R-project . org 